\title{
Thermodynamics of scalar-tensor theory with non-minimally derivative coupling
}

\author{
Yumei Huang, ${ }^{1, \mathrm{a}}$, Yungui Gong ${ }^{2, \mathrm{~b}}$, Dicong Liang ${ }^{3, \mathrm{c}}$, Zhu Yi ${ }^{3, \mathrm{~d}}$ \\ ${ }^{1}$ Department of Astronomy, Beijing Normal University, Beijing 100875, China \\ ${ }^{2}$ MOE Key Laboratory of Fundamental Quantities Measurement, School of Physics, Huazhong University of Science and Technology, \\ Wuhan 430074, Hubei, China \\ ${ }^{3}$ School of Physics, Huazhong University of Science and Technology, Wuhan 430074, Hubei, China
}

Received: 6 April 2015 / Accepted: 19 July 2015 / Published online: 30 July 2015

(C) The Author(s) 2015. This article is published with open access at Springerlink.com

\begin{abstract}
With the usual definitions for the entropy and the temperature associated with the apparent horizon, we show that the unified first law on the apparent horizon is equivalent to the Friedmann equation for the scalar-tensor theory with non-minimally derivative coupling. The second law of thermodynamics on the apparent horizon is also satisfied. The results support a deep and fundamental connection between gravitation, thermodynamics, and quantum theory.
\end{abstract}

\section{Introduction}

The discovery of black hole thermodynamics [1] has shown a deep connection between gravitation and thermodynamics. In particular, the black hole temperature, which is proportional to the surface gravity at the event horizon, and Hawking radiation [2] tell us that this relation may be linked to quantum gravity [3]. Instead of being proportional to the volume, the Bekenstein-Hawking entropy is equal to one quarter of the area of the event horizon of the black hole measured in Planck units [2,4]. Based on this area law of entropy, Bekenstein then argued for a universal entropy bound for a weakly self-gravitating physical system in an asymptotically flat space-time [5]. This led to the proposal of the holographic principle [6-8]. The holographic principle was supported by the AdS/CFT correspondence, which states that the type IIB superstring theory on $\mathrm{AdS}_{5} \times S^{5}$ is equivalent to the $N=4$ super-Yang-Mills theory with gauge group $U(N)$ in four dimensions [9]. The AdS/CFT correspondence relates a gravitational theory in $d$-dimensional anti-de Sitter space with a

\footnotetext{
a e-mail: huangymei@gmail.com

be-mail: yggong@mail.hust.edu.cn

c e-mail: 904186306@qq.com

de-mail: yizhuhust@sina.cn
}

conformal field theory living in a $(d-1)$-dimensional boundary space. The Hawking radiation and the holography show that the thermodynamic property of gravitation is unique. These special properties may provide some physical insights into the nature of quantum gravity. By applying the area law of entropy for all local acceleration horizons, it was found that the Einstein equation could be derived from the first law of thermodynamics [10]. The relation was then discussed in cosmology, and the equivalence between the first law of thermodynamics and the Friedmann equation was derived [1115]. The relation between thermodynamics and gravitation was discussed extensively in the literature, and the relation holds also in more general theories of gravity [10-32].

The simplest generalization of Einstein's general relativity is Brans-Dicke theory [33]. In Brans-Dicke theory, gravitation is propagated by massless spin zero scalar field in addition to the massless spin 2 graviton. The scalar degree of freedom can also arise upon compactification of higher dimensions. In general, the scalar field $\phi$ is coupled to the curvature scalar $R$ as $f(\phi) R$. More general couplings for the scalar field are also possible [34-37]. In Horndeski theory, the derivatives of both the metric $g_{\mu \nu}$ and the scalar field $\phi$ are at most second order, and the second derivative $\phi_{; \mu \nu}$ couples to the Einstein tensor by a term of the general form $f(\phi, X) G^{\mu v} \phi_{; \mu \nu}$, where $X=g^{\mu v} \phi_{, \mu} \phi_{, v}$ [34]. However, the field equations are still second order in Horndeski theory. We can also consider the non-minimally derivative coupling $\phi_{, \mu}$ $\phi^{, \mu} R, \phi_{, \mu} \phi_{, \nu} R^{\mu \nu}, \phi \square \phi R, \phi \phi_{; \mu \nu} R^{\mu \nu}, \phi \phi_{, \mu} R^{; \mu}$, and $\phi^{2} \square R$. If we choose the non-minimally derivative coupling as $G^{\mu \nu} \phi_{, \mu} \phi_{, v}$, then the field equations contain no more than second derivatives [38], and the theory avoids the BoulwareDeser ghost [39]. With this choice of non-minimally derivative coupling, it was shown that the Higgs field produced a successful slow-roll inflation without violating the unitarity bound and fine-tuning the coupling constant $\lambda[40]$. 
The scalar-tensor theory with the non-minimally derivative coupling $\omega^{2} G^{\mu \nu} \phi_{, \mu} \phi_{, v}$ was discussed by lots of researchers recently [41-70].

In this paper, we discuss the thermodynamics of the scalar-tensor theory with non-minimally derivative coupling $\omega^{2} G^{\mu \nu} \phi_{, \mu} \phi_{, v}$. The paper is organized as follows. In Sect. 2, we review the scalar-tensor theory with non-minimally derivative coupling. The relation between the first law of thermodynamics and the Friedmann equation is presented in Sect. 3. We discuss the second law of thermodynamics in Sect. 4, and conclusions are drawn in Sect. 5.

\section{The scalar-tensor theory with non-minimally derivative coupling}

The action for the general scalar-tensor theory with nonminimally derivative coupling which contains only up to second derivatives is

$$
\begin{aligned}
S= & \int \mathrm{d}^{4} x \sqrt{-g}\left[\frac{M_{\mathrm{pl}}^{2}}{2} R\right. \\
& \left.-\frac{1}{2}\left(g^{\mu \nu}-\omega^{2} G^{\mu \nu}\right) \partial_{\mu} \phi \partial_{\nu} \phi-V(\phi)\right]+S_{b},
\end{aligned}
$$

where the Planck mass $M_{\mathrm{pl}}^{2}=(8 \pi G)^{-1}=\kappa^{-2}, w$ is the coupling constant with the dimension of inverse mass, $V(\phi)$ corresponds to the scalar field potential, and $S_{b}$ is the action for the background matter, which includes dust and radiation. Varying the action (1) with respect to the metric $g_{\mu \nu}$, we get

$G_{\mu \nu}=R_{\mu \nu}-\frac{1}{2} g_{\mu \nu} R=\kappa^{2}\left(T_{\mu \nu}^{b}+T_{\mu \nu}^{c}\right)$,

where $T_{\mu \nu}^{b}$ is the energy-momentum tensor for the background matter, and the effective energy-momentum tensor $T_{\mu \nu}^{c}$ for the scalar field is

$$
\begin{aligned}
T_{\mu \nu}^{c}= & \phi_{, \mu} \phi_{, \nu}-\frac{1}{2} g_{\mu \nu}\left(\phi_{, \alpha}\right)^{2}-g_{\mu \nu} V(\phi) \\
& -\omega^{2}\left\{-\frac{1}{2} \phi_{, \mu} \phi_{, \nu} R+2 \phi_{, \alpha} \nabla_{(\mu} \phi R_{\nu)}^{\alpha}+\phi^{, \alpha} \phi^{, \beta} R_{\mu \alpha \nu \beta}\right. \\
& +\nabla_{\mu} \nabla^{\alpha} \phi \nabla_{\nu} \nabla_{\alpha} \phi-\nabla_{\mu} \nabla_{\nu} \phi \square \phi-\frac{1}{2}\left(\phi_{, \alpha}\right)^{2} G_{\mu \nu} \\
& \left.+g_{\mu \nu}\left[-\frac{1}{2} \nabla^{\alpha} \nabla^{\beta} \phi \nabla_{\alpha} \nabla_{\beta} \phi+\frac{1}{2}(\square \phi)^{2}-\phi_{, \alpha} \phi_{, \beta} R^{\alpha \beta}\right]\right\} .
\end{aligned}
$$

Using the homogeneous and isotropic Friedmann-RobertsonWalker (FRW) metric,

$$
\mathrm{d} s^{2}=-\mathrm{d} t^{2}+\frac{a(t)^{2}}{1-k r^{2}} \mathrm{~d} r^{2}+a(t)^{2} r^{2}\left(\mathrm{~d} \theta^{2}+\sin ^{2} \theta \mathrm{d} \phi^{2}\right)
$$

where $k=0,-1,+1$ represents a flat, open, and closed universe respectively, we obtain $T_{00}^{c}$ and $T_{11}^{c}$ :

$$
\begin{aligned}
T_{00}^{c}= & \frac{1}{2} \dot{\phi}^{2}+V(\phi)+\frac{9}{2} \omega^{2} H^{2} \dot{\phi}^{2}+\frac{3}{2} \omega^{2} \frac{k}{a^{2}} \dot{\phi}^{2}, \\
T_{11}^{c}= & \frac{a^{2}}{1-k r^{2}}\left[\frac{1}{2} \dot{\phi}^{2}-V(\phi)\right. \\
& \left.-\frac{\omega^{2}}{2} \dot{\phi}^{2}\left(2 \dot{H}+3 H^{2}-\frac{k}{a^{2}}+\frac{4 H \ddot{\phi}}{\dot{\phi}}\right)\right] .
\end{aligned}
$$

Therefore, the effective energy density and pressure for the scalar field are given by

$$
\begin{aligned}
& \rho_{c}=\frac{\dot{\phi}^{2}}{2}\left(1+9 \omega^{2} H^{2}+3 \omega^{2} \frac{k}{a^{2}}\right)+V(\phi), \\
& p_{c}=\frac{1}{2} \dot{\phi}^{2}-V(\phi)-\frac{\omega^{2}}{2} \dot{\phi}^{2}\left(2 \dot{H}+3 H^{2}-\frac{k}{a^{2}}+\frac{4 H \ddot{\phi}}{\dot{\phi}}\right) .
\end{aligned}
$$

The Friedman equations are

$$
\begin{aligned}
H^{2}+\frac{k}{a^{2}}= & \frac{8 \pi G}{3}\left(\rho_{b}+\rho_{c}\right)=\frac{8 \pi G}{3}\left[\frac{1}{2} \dot{\phi}^{2}+V(\phi)\right. \\
& \left.+\frac{9}{2} \omega^{2} H^{2} \dot{\phi}^{2}+\frac{3}{2} \omega^{2} \frac{k}{a^{2}} \dot{\phi}^{2}+\rho_{b}\right], \\
\dot{H}-\frac{k}{a^{2}}= & -4 \pi G\left[\dot{\phi}^{2}+3 \omega^{2} H^{2} \dot{\phi}^{2}\right. \\
& \left.+2 \omega^{2} \frac{k}{a^{2}} \dot{\phi}^{2}-\omega^{2} \frac{\mathrm{d}}{\mathrm{d} t}\left(H \dot{\phi}^{2}\right)+\rho_{b}+p_{b}\right] .
\end{aligned}
$$

If the non-minimally derivative coupling is absent, $\omega^{2}=$ 0 , we recover the standard result of Einstein gravity with canonically scalar field.

\section{The relation between the first law of thermodynamics and Friedmann equation}

In this section, we discuss the equivalence between the first law of thermodynamics on the apparent horizon and Friedmann equation. For a spherically symmetric space-time with the metric $\mathrm{d} s^{2}=g_{a b} \mathrm{~d} x^{a} \mathrm{~d} x^{b}+\tilde{r}^{2} \mathrm{~d} \Omega^{2}$, where the unit spherical metric $\mathrm{d} \Omega^{2}=\mathrm{d} \theta^{2}+\sin ^{2} \theta \mathrm{d} \phi^{2}$, the apparent horizon is defined as $f=g^{a b} \tilde{r}_{, a} \tilde{r}_{, b}=0$, the dynamical surface gravity at the apparent horizon is $\kappa=\nabla_{a} \nabla^{a} \tilde{r} / 2$ [16], and the Hawking temperature associated with the apparent horizon is $T=|\kappa| / 2 \pi$. For the FRW metric (4), the apparent horizon is

$\tilde{r}_{A}=\left(H^{2}+k / a^{2}\right)^{-1 / 2}$.

The surface gravity at the apparent horizon is

$\kappa=\frac{1}{2} \nabla_{a} \nabla^{a} \tilde{r}=-\frac{1}{\tilde{r}_{A}}\left(1-\frac{\dot{\tilde{r}}_{A}}{2 H \tilde{r}_{A}}\right)$, 
and the associated temperature is $T_{A}=\kappa / 2 \pi$. The entropy enclosed by the apparent horizon is $S_{A}=\pi \tilde{r}_{A}^{2} / G$. Therefore, we have

$$
\begin{aligned}
T_{A} \mathrm{~d} S_{A} & =-\frac{1}{2 \pi \tilde{r}_{A}}\left(1-\frac{\dot{\tilde{r}}_{A}}{2 H \tilde{r}_{A}}\right) \frac{2 \pi \tilde{r}_{A}}{G} \dot{\tilde{r}}_{A} \mathrm{~d} t \\
& =-\frac{1}{G}\left(1-\frac{\dot{\tilde{r}}_{A}}{2 H \tilde{r}_{A}}\right) \dot{\tilde{r}}_{A} \mathrm{~d} t .
\end{aligned}
$$

For the scalar-tensor theory with non-minimally derivative coupling, the effective total energy density is $\rho_{\text {tot }}=$ $\rho_{b}+\rho_{c}$. The total energy of the system inside the apparent horizon is $E=\rho_{\text {tot }} V$, where the volume $V=4 \pi \tilde{r}_{A}^{3} / 3$. So the energy change is

$\mathrm{d} E=\rho_{\text {tot }} \mathrm{d} V+V \mathrm{~d} \rho_{\text {tot }}=\rho_{\text {tot }} 4 \pi \tilde{r}_{A}^{2} \mathrm{~d} \tilde{r}_{A}+\frac{4}{3} \pi \tilde{r}_{A}^{3} \mathrm{~d} \rho_{\text {tot }}$,

where $\mathrm{d} \tilde{r}_{A}=\dot{\tilde{r}}_{A} \mathrm{~d} t$, and $\mathrm{d} \rho_{\text {tot }}=\dot{\rho}_{\text {tot }} \mathrm{d} t$. By using the energy conservation for the total energy,

$\dot{\rho}_{\text {tot }}+3 H\left(\rho_{\text {tot }}+p_{\text {tot }}\right)=0$,

we have

$\mathrm{d} \rho_{\text {tot }}=-3 H\left(\rho_{\text {tot }}+p_{\text {tot }}\right) \mathrm{d} t$.

Substituting the above result into Eq. (14), we get

$\mathrm{d} E=4 \pi \tilde{r}_{A}^{2} \rho_{\mathrm{tot}} \dot{\tilde{r}}_{A} \mathrm{~d} t-4 \pi \tilde{r}_{A}^{3} H\left(\rho_{\mathrm{tot}}+p_{\mathrm{tot}}\right) \mathrm{d} t$.

The work term $W \mathrm{~d} V$ with $W=\left(\rho_{\text {tot }}-p_{\text {tot }}\right) / 2$ is

$W \mathrm{~d} V=2 \pi \tilde{r}_{A}^{2}\left(\rho_{\text {tot }}-p_{\text {tot }}\right) \dot{\tilde{r}}_{A} \mathrm{~d} t$

Applying the unified first law,

$\mathrm{d} E=T_{A} \mathrm{~d} S_{A}+W \mathrm{~d} V$

we get

$4 \pi \tilde{r}_{A}^{3} H\left(\rho_{\text {tot }}+p_{\text {tot }}\right)\left(1-\frac{\dot{\tilde{r}}_{A}}{2 H \tilde{r}_{A}}\right)=\frac{1}{G}\left(1-\frac{\dot{\tilde{r}}_{A}}{2 H \tilde{r}_{A}}\right) \dot{\tilde{r}}_{A}$.

Taking the time derivative of the apparent horizon $\tilde{r}_{A}$ defined in Eq. (11), we get

$\dot{\tilde{r}}_{A}=-\tilde{r}_{A}^{3} H\left(\dot{H}-\frac{k}{a^{2}}\right)$
Combining Eqs. (20) and (21), we get

$$
\begin{aligned}
\dot{H}-\frac{k}{a^{2}}= & -4 \pi G\left(\rho_{\text {tot }}+p_{\text {tot }}\right)=-4 \pi G\left[\dot{\phi}^{2}+3 \omega^{2} H^{2} \dot{\phi}^{2}\right. \\
& \left.+2 \omega^{2} \frac{k}{a^{2}} \dot{\phi}^{2}-\omega^{2} \frac{\mathrm{d}}{\mathrm{d} t}\left(H \dot{\phi}^{2}\right)+\rho_{b}+p_{b}\right] .
\end{aligned}
$$

Using the energy conservation equation (15), and integrating Eq. (22), we obtain the Friedman equation

$H^{2}+\frac{k}{a^{2}}=\frac{8 \pi G}{3}\left[\frac{\dot{\phi}^{2}}{2}\left(1+9 \omega^{2} H^{2}+3 \omega^{2} \frac{k}{a^{2}}\right)+V(\phi)+\rho_{b}\right]$.

Thus, we derive the Friedmann equation from the unified first law on the apparent horizon for the scalar-tensor theory with non-minimally derivative coupling.

Now we would like to derive the unified first law starting from the Friedmann equation. Substituting Eq. (10) into Eq. (21), we obtain

$$
\begin{aligned}
\mathrm{d} \tilde{r}_{A}= & 4 \pi G H \tilde{r}_{A}^{3}\left[\dot{\phi}^{2}+3 \omega^{2} H^{2} \dot{\phi}^{2}+2 \omega^{2} \frac{k}{a^{2}} \dot{\phi}^{2}\right. \\
& \left.-\omega^{2} \frac{\mathrm{d}}{\mathrm{d} t}\left(H \dot{\phi}^{2}\right)+\rho_{b}+p_{b}\right] \mathrm{d} t .
\end{aligned}
$$

We multiply by $-\left[1-\dot{\tilde{r}}_{A} /\left(2 H \tilde{r}_{A}\right)\right] / G$ both sides of Eq. (24); then Eq. (24) becomes

$$
\begin{aligned}
T_{A} \mathrm{~d} S_{A}= & -\frac{1}{2 \pi \tilde{r}_{A}}\left(1-\frac{\dot{\tilde{r}}_{A}}{2 H \tilde{r}_{A}}\right) \cdot d\left(\frac{4 \pi \tilde{r}_{A}^{2}}{4 G}\right) \\
= & -4 \pi H\left[\dot{\phi}^{2}+3 \omega^{2} H^{2} \dot{\phi}^{2}+2 \omega^{2} \frac{k}{a^{2}} \dot{\phi}^{2}\right. \\
& \left.-\omega^{2} \frac{\mathrm{d}}{\mathrm{d} t}\left(H \dot{\phi}^{2}\right)+\rho_{b}+p_{b}\right] \tilde{r}_{A}^{3}\left(1-\frac{\dot{\tilde{r}}_{A}}{2 H \tilde{r}_{A}}\right) \mathrm{d} t \\
= & -4 \pi H\left(\rho_{\text {tot }}+p_{\text {tot }}\right) \tilde{r}_{A}^{3}\left(1-\frac{\dot{\tilde{r}}_{A}}{2 H \tilde{r}_{A}}\right) \mathrm{d} t
\end{aligned}
$$

Combining Eq. (25) with Eq. (18), we get

$$
\begin{aligned}
T_{A} \mathrm{~d} S_{A}+W \mathrm{~d} V & =-4 \pi \tilde{r}_{A}^{3} H\left(\rho_{\text {tot }}+p_{\text {tot }}\right) \mathrm{d} t+4 \pi \tilde{r}_{A}^{2} \rho_{\text {tot }} \dot{\tilde{r}}_{A} \mathrm{~d} t \\
& =\frac{4 \pi}{3} \tilde{r}_{A}^{3} \mathrm{~d} \rho_{\text {tot }}+4 \pi \rho_{\text {tot }} \tilde{r}_{A}^{2} \mathrm{~d} \tilde{r}_{A}=\mathrm{d} E .
\end{aligned}
$$

So the unified first law is derived from the Friedmann equation together with the energy conservation equation. Thus, with the usual definitions for the entropy and the Hawking temperature associated with the apparent horizon, we show that the unified first law on the apparent horizon is equivalent to the Friedmann equation for the scalar-tensor theory with non-minimally derivative coupling. 


\section{The second law of thermodynamics on the apparent horizon}

As discussed in the previous section, the entropy of the apparent horizon is $S_{A}=A /(4 G)=\pi \tilde{r}_{A}^{2} / G$, so

$\dot{S}_{A}=\frac{2 \pi \tilde{r}_{A}}{G} \dot{\tilde{r}}_{A}=-\frac{2 \pi \tilde{r}_{A}^{4}}{G} H\left(\dot{H}-\frac{k}{a^{2}}\right)$.

By using the Friedmann equations (9) and (10), we get

$$
\begin{aligned}
\dot{S}_{A} & =3 S_{A} H \frac{\dot{\phi}^{2}+3 \omega^{2} H^{2} \dot{\phi}^{2}+2 \omega^{2} k \dot{\phi}^{2} / a^{2}-\omega^{2} \frac{\mathrm{d}}{\mathrm{d} t}\left(H \dot{\phi}^{2}\right)+\rho_{b}+p_{b}}{\dot{\phi}^{2}\left(1+9 \omega^{2} H^{2}+3 \omega^{2} k / a^{2}\right) / 2+V(\phi)+\rho_{b}} \\
& =3 S_{A} H \frac{\rho_{\text {tot }}+p_{\text {tot }}}{\rho_{\text {tot }}} .
\end{aligned}
$$

As long as $\rho_{\text {tot }}+p_{\text {tot }} \geq 0$, we have $\dot{S}_{A} \geq 0$, and the second law of thermodynamics on the apparent horizon is satisfied.

\section{Conclusions}

With the usual definition of the area law of entropy $S_{A}=$ $\pi \tilde{r}_{A}^{2} /(4 G)$ of the apparent horizon, and the temperature $T_{A}=-\left[1-\dot{\tilde{r}}_{A} /\left(2 H \tilde{r}_{A}\right)\right] /\left(2 \pi \tilde{r}_{A}\right)$, as well as the energy conservation for the effective total energy density $\dot{\rho}_{\text {tot }}+$ $3 H\left(\rho_{\text {tot }}+p_{\text {tot }}\right)=0$, we show that the unified first law of thermodynamics, $\mathrm{d} E=T_{A} \mathrm{~d} S_{A}+W \mathrm{~d} V$, is equivalent to the Friedmann equation for the scalar-tensor theory with non-minimally derivative coupling. The result further supports the argument that the apparent horizon is a physical boundary and the relation between the first law of thermodynamics and Friedmann equation holds for a more general theory of gravity and suggests a deep and fundamental connection between gravitation, thermodynamics, and quantum theory. Furthermore, we show that the second law of thermodynamics on the apparent horizon is also satisfied for the scalar-tensor theory with non-minimally derivative coupling as long as $\rho_{\text {tot }}+p_{\text {tot }} \geq 0$.

Acknowledgments This research was supported in part by the Natural Science Foundation of China under Grant Nos. 11175270 and 11475065 , and the Program for New Century Excellent Talents in University under Grant No. NCET-12-0205.

Open Access This article is distributed under the terms of the Creative Commons Attribution 4.0 International License (http://creativecomm ons.org/licenses/by/4.0/), which permits unrestricted use, distribution, and reproduction in any medium, provided you give appropriate credit to the original author(s) and the source, provide a link to the Creative Commons license, and indicate if changes were made. Funded by SCOAP ${ }^{3}$.

\section{References}

1. J.M. Bardeen, B. Carter, S. Hawking, Commun. Math. Phys. 31, 161 (1973). doi:10.1007/BF01645742
2. S. Hawking, Commun. Math. Phys. 43, 199 (1975). doi:10.1007/ BF02345020

3. R.M. Wald, Living Rev. Relativ. 4, 6 (2001)

4. J.D. Bekenstein, Phys. Rev. D 7, 2333 (1973). doi:10.1103/ PhysRevD.7.2333

5. J.D. Bekenstein, Phys. Rev. D 23, 287 (1981). doi:10.1103/ PhysRevD.23.287

6. G. 't Hooft, Salamfest pp. 0284-296 (1993)

7. L. Susskind, J. Math. Phys. 36, 6377 (1995). doi:10.1063/1.531249

8. E. Witten, Adv. Theor. Math. Phys. 2, 253 (1998)

9. J.M. Maldacena, Adv. Theor. Math. Phys. 2, 231 (1998)

10. T. Jacobson, Phys. Rev. Lett. 75, 1260 (1995). doi:10.1103/ PhysRevLett.75.1260

11. R.G. Cai, S.P. Kim, JHEP 0502, 050 (2005). doi:10.1088/ 1126-6708/2005/02/050

12. M. Akbar, R.G. Cai, Phys. Rev. D 75, 084003 (2007). doi:10.1103/ PhysRevD.75.084003

13. R.G. Cai, L.M. Cao, Phys. Rev. D 75, 064008 (2007). doi:10.1103/ PhysRevD.75.064008

14. Y. Gong, B. Wang, A. Wang, JCAP 0701, 024 (2007). doi:10.1088/ 1475-7516/2007/01/024

15. Y. Gong, A. Wang, Phys. Rev. Lett. 99, 211301 (2007). doi:10. 1103/PhysRevLett.99.211301

16. S.A. Hayward, Class. Quantum Gravity 15, 3147 (1998). doi:10. 1088/0264-9381/15/10/017

17. T. Padmanabhan, Class. Quantum Gravity 19, 5387 (2002). doi:10. 1088/0264-9381/19/21/306

18. T. Padmanabhan, Phys. Rep. 406, 49 (2005). doi:10.1016/j. physrep.2004.10.003

19. B. Wang, Y. Gong, E. Abdalla, Phys. Rev. D 74, 083520 (2006). doi:10.1103/PhysRevD.74.083520

20. C. Eling, R. Guedens, T. Jacobson, Phys. Rev. Lett. 96, 121301 (2006). doi:10.1103/PhysRevLett.96.121301

21. D. Kothawala, S. Sarkar, T. Padmanabhan, Phys. Lett. B 652, 338 (2007). doi:10.1016/j.physletb.2007.07.021

22. J. Zhou, B. Wang, Y. Gong, E. Abdalla, Phys. Lett. B 652, 86 (2007). doi:10.1016/j.physletb.2007.06.067

23. Y. Gong, B. Wang, A. Wang, Phys. Rev. D 75, 123516 (2007). doi:10.1103/PhysRevD.75.123516

24. R.G. Cai, L.M. Cao, Y.P. Hu, Class. Quantum Gravity 26, 155018 (2009). doi:10.1088/0264-9381/26/15/155018

25. S. Hayward, R. Di Criscienzo, L. Vanzo, M. Nadalini, S. Zerbini, Class. Quantum Gravity 26, 062001 (2009). doi:10.1088/ 0264-9381/26/6/062001

26. T. Padmanabhan, Rep. Prog. Phys. 73, 046901 (2010). doi:10.1088/ 0034-4885/73/4/046901

27. M. Sharif, M. Zubair, JCAP 1203, 028 (2012). doi:10.1088/ 1475-7516/2012/05/E01,10.1088/1475-7516/2012/03/028

28. M. Sharif, M. Zubair, JCAP 1311, 042 (2013). doi:10.1088/ 1475-7516/2013/11/042

29. T. Padmanabhan, Gen. Relativ. Gravit. 46, 1673 (2014). doi:10. 1007/s10714-014-1673-7

30. P. Bintruy, A. Helou, The Apparent Universe (2014). arXiv: 1406.1658

31. S. Mitra, S. Saha, S. Chakraborty, Adv. High Energy Phys. 2015, 430764 (2015). doi:10.1155/2015/430764

32. A. Helou, Dynamics of the cosmological apparent horizon: surface gravity \& temperature (2015). arXiv: 1502.04235

33. C. Brans, R. Dicke, Phys. Rev. 124, 925 (1961). doi:10.1103/ PhysRev.124.925

34. G.W. Horndeski, Int. J. Theor. Phys. 10, 363 (1974). doi:10.1007/ BF01807638

35. L. Amendola, Phys. Lett. B 301, 175 (1993). doi:10.1016/ 0370-2693(93)90685-B

36. S. Capozziello, G. Lambiase, Gen. Relativ. Gravit. 31, 1005 (1999). doi:10.1023/A:1026631531309 
37. S. Capozziello, G. Lambiase, H. Schmidt, Ann. Phys. 9, 39 (2000)

38. S.V. Sushkov, Phys. Rev. D 80, 103505 (2009). doi:10.1103/ PhysRevD.80.103505

39. D. Boulware, S. Deser, Phys. Lett. B 40, 227 (1972). doi:10.1016/ 0370-2693(72)90418-2

40. C. Germani, A. Kehagias, Phys. Rev. Lett. 105, 011302 (2010). doi:10.1103/PhysRevLett.105.011302

41. S.F. Daniel, R.R. Caldwell, Class. Quantum Gravity 24, 5573 (2007). doi:10.1088/0264-9381/24/22/017

42. E.N. Saridakis, S.V. Sushkov, Phys. Rev. D 81, 083510 (2010). doi:10.1103/PhysRevD.81.083510

43. S. Sushkov, Phys. Rev. D 85, 123520 (2012). doi:10.1103/ PhysRevD.85.123520

44. M.A. Skugoreva, S.V. Sushkov, A.V. Toporensky, Phys. Rev. D 88(10), 083539 (2013). doi:10.1103/PhysRevD.88.109906, 10. 1103/PhysRevD.88.083539

45. C. Germani, A. Kehagias, JCAP 1005, 019 (2010). doi:10.1088/ 1475-7516/2010/05/019, 10.1088/1475-7516/2010/06/E01

46. C. Germani, Y. Watanabe, JCAP 1107, 031 (2011). doi:10.1088/ 1475-7516/2011/07/031, 10.1088/1475-7516/2011/07/A01

47. A. De Felice, S. Tsujikawa, Phys. Rev. D 84, 083504 (2011). doi:10. 1103/PhysRevD.84.083504

48. S. Tsujikawa, Phys. Rev. D 85, 083518 (2012). doi:10.1103/ PhysRevD.85.083518

49. H.M. Sadjadi, Phys. Rev. D 83, 107301 (2011). doi:10.1103/ PhysRevD.83.107301

50. H.M. Sadjadi, Gen. Relativ. Gravit. 46(11), 1817 (2014). doi:10. 1007/s10714-014-1817-9

51. M. Minamitsuji, Phys. Rev. D 89(6), 064017 (2014). doi:10.1103/ PhysRevD.89.064017

52. L. Granda, JCAP 1007, 006 (2010). doi:10.1088/1475-7516/2010/ 07/006

53. L. Granda, W. Cardona, JCAP 1007, 021 (2010). doi:10.1088/ $1475-7516 / 2010 / 07 / 021$
54. L. Granda, Mod. Phys. Lett. A 27, 1250018 (2012). doi:10.1142/ S0217732312500186

55. R. Jinno, K. Mukaida, K. Nakayama, JCAP 1401, 031 (2014). doi:10.1088/1475-7516/2014/01/031

56. M. Sami, M. Shahalam, M. Skugoreva, A. Toporensky, Phys. Rev. D 86, 103532 (2012). doi:10.1103/PhysRevD.86.103532

57. A. Anabalon, A. Cisterna, J. Oliva, Phys. Rev. D 89(8), 084050 (2014). doi:10.1103/PhysRevD.89.084050

58. M. Rinaldi, Phys. Rev. D 86, 084048 (2012). doi:10.1103/ PhysRevD.86.084048

59. G. Koutsoumbas, K. Ntrekis, E. Papantonopoulos, JCAP 1308, 027 (2013). doi:10.1088/1475-7516/2013/08/027

60. A. Cisterna, C. Erices, Phys. Rev. D 89(8), 084038 (2014). doi:10. 1103/PhysRevD.89.084038

61. Y. Huang, Q. Gao, Y. Gong, Eur. J. Phys. C 75, 143 (2015). doi:10. 1140/epjc/s10052-015-3365-1

62. M. Bravo-Gaete, M. Hassaine, Phys. Rev. D 89(10), 104028 (2014). doi:10.1103/PhysRevD.89.104028

63. M. Bravo-Gaete, M. Hassaine, Phys. Rev. D 90(2), 024008 (2014). doi:10.1103/PhysRevD.90.024008

64. J.P. Bruneton, M. Rinaldi, A. Kanfon, A. Hees, S. Schlogel et al., Adv. Astron. 2012, 430694 (2012). doi:10.1155/2012/430694

65. K. Feng, T. Qiu, Y.S. Piao, Phys. Lett. B 729, 99 (2014). doi:10. 1016/j.physletb.2014.01.008

66. K. Feng, T. Qiu, Phys. Rev. D 90(12), 123508 (2014). doi:10.1103/ PhysRevD.90.123508

67. I. Dalianis, F. Farakos, Phys. Lett. B 736, 299 (2014). doi:10.1016/ j.physletb.2014.07.028

68. I. Dalianis, F. Farakos, Phys. Rev. D 90(8), 083512 (2014). doi:10. 1103/PhysRevD.90.083512

69. I. Dalianis, F. Farakos, Non-minimal derivative couplings and inflation in supergravity (2015). arXiv: 1504.06875

70. N. Yang, Q. Gao, Y. Gong, Inflationary models with non-minimally derivative coupling (2015). arXiv:1504.05839 